\title{
Technical note: Bovine adipocyte and preadipocyte co-culture as an efficient adipogenic model
}

\author{
Clarissa Strieder-Barboza, ${ }^{*}$ Eileen Thompson, Kyan Thelen, and G. Andres Contreras $†$ \\ Department of Large Animal Clinical Sciences, Michigan State University, East Lansing 48824
}

\section{ABSTRACT}

Reductionist studies of adipose tissue biology require reliable in vitro adipocyte culturing models. Current protocols for adipogenesis induction in stromal vascular fraction-derived preadipocytes require extended culturing periods and have low adipogenic rates. We compared the adipogenic efficiency of a 7-d co-culture model of visceral (VIS) and subcutaneous (SC) stromal vascular fraction-derived preadipocytes with mature adipocytes with a 14-d standard adipocyte differentiation protocol. We obtained preadipocytes and mature adipocytes from SC and VIS adipose tissue of nonlactating, nongestating Holstein cows $(\mathrm{n}=6)$. Adipogenesis induction was performed using a standard protocol for 7 (SD7; control) or $14 \mathrm{~d}$ (SD14), and a co-culture model for $7 \mathrm{~d}$ (CC7). Culture conditions, including medium composition, were the same for all treatments. For CC7, 900 primary adipocytes $/ \mathrm{cm}^{2}$ were placed in $0.4-\mu \mathrm{m}$ transwell inserts and co-cultured with preadipocytes for adipogenesis induction. Both CC7 and SD14 similarly stimulated gene expression of adipogenic genes such as $A D I P O Q, C E B P A$, and $C E B P B$ in VIS and $\mathrm{SC}$. The CC7 increased triacylglycerol accumulation compared with SD14 and SD7. CC7 augmented triacylglycerol accumulation by $40-$ and 16 -fold in SC and VIS compared with 22- and 4-fold increment in SD14, respectively. Lipolytic responses to 2 -h $\beta$-adrenergic stimulation with $1 \mu M$ isoproterenol were higher in CC7 and SD14 than SD7 in SC; CC7 increased glycerol release compared with SD7 in VIS but SD7 and SD14 had similar responses. Overall, CC7 was more efficient in inducing adipogenesis in preadipocytes from VIS and SC than SD14. Furthermore, CC7 stimulated similar lipolysis and lipogenic responses than SD14 but in a shorter time. The adipogenic approach of co-culturing preadipocytes with mature adipocytes will improve the

Received August 30, 2018.

Accepted December 14, 2018.

*Current affiliation: Department of General Surgery, University of Michigan Medical School, Ann Arbor, MI, 48109

†Corresponding author: contre28@msu.edu use of reductionist models to study adipocyte physiology in dairy cows and the assessment of pharmacological or nutritional interventions for enhancing dairy cow health and production.

Key words: adipocytes, adipogenesis, bovine, coculture, in vitro

\section{Technical Note}

Dairy cows rely on adipocyte triacylglycerol (TAG) reserves to offset periparturient negative energy balance and ensure milk production throughout lactation (Contreras et al., 2018). The use of reductionist approaches, such as in vitro adipocyte culturing, is necessary to understand the adipose tissue (AT) biology and to assess novel of pharmacological or nutritional interventions in dairy cows. Primary adipocyte isolation and culturing is difficult because these cells are buoyant and lack sufficient matrix protein secretion to facilitate in vitro adherence (Sugihara et al., 1987; Picot et al., 2005). An alternate approach is to obtain preadipocytes from stromal vascular fraction (SVF) and induce adipogenesis. Adipogenesis is defined as the process of differentiation of preadipocytes into adipocytes. Across species, a standard way to induce in vitro adipogenesis is through their exposure to medium containing a mixture of hormones, such as glucocorticoids, agents to increase cyclic AMP activity, and insulin (MacDougald and Mandrup, 2002). In addition, bovine adipocyte differentiation medium is supplemented with unbound fatty acids, such as acetate and octanoate, bovine serum lipids, and peroxisome proliferator activated receptor gamma ligands, such as rosiglitazone and troglitazone (Grant et al., 2008; Smith et al., 2009; Lengi and Corl, 2010). Among the main disadvantages of the standard differentiation are the length of protocols $(14-21 \mathrm{~d})$ and the low adipogenesis efficiency, mainly with visceral (VIS) SVF-derived preadipocytes. Subcutaneous (SC) preadipocytes are more committed to differentiate, whereas those from VIS depots have an antiadipogenic profile and require growth factors to undergo differentiation (Macotela et al., 2012). As a result, information on VIS-specific regulatory mecha- 
nisms and adipokine secretion in bovine is limited. Coculture (CC) is an alternative model for adipogenesis induction, allowing preadipocytes to differentiate in the presence of mature adipocytes. The direct $\mathrm{CC}$ of human primary adipocytes with preadipocytes for 16 $\mathrm{d}$ was more effective inducing adipogenesis compared with standard differentiation, as it induced the expression of adipogenic markers to levels observed in freshly isolated adipocytes and released more glycerol, leptin, and adiponectin when compared with standard differentiation (Stacey et al., 2009). These results suggested that, although standard differentiation is currently the most common method for achieving adipogenesis in vitro, it may be less efficient than CC (Stacey et al., 2009). Besides Stacey et al. (2009), other studies have suggested that mature adipocytes in $\mathrm{CC}$ release growth factors that enhance preadipocyte differentiation and better mimic the adipogenic stimuli provided in vivo (Considine et al., 1996). However, other mechanisms underlying the increased adipogenic induction in CC remain to be established. The goal of our study was to compare the efficiency of 2 culture conditions for adipogenesis induction: CC of preadipocytes with mature adipocytes and the standard differentiation. We hypothesized that CC model enhances adipogenesis in bovine adipocytes compared with standard differentiation.

Samples of SC and VIS AT from flank and omentum, respectively, were obtained from 6 multiparous, nonlactating, nongestating Holstein cows at a local abattoir. The SVF was isolated as described previously (Strieder-Barboza et al., 2018). Briefly, AT samples were transported to the laboratory while in KrebsRinger bicarbonate buffer (KRBB; $135 \mathrm{~m} M \mathrm{NaCl}, 5$ $\mathrm{m} M \mathrm{KCl}, 1 \mathrm{~m} M \mathrm{MgSO}_{4}, 0.4 \mathrm{~m} M \mathrm{~K}_{2} \mathrm{HPO}_{4}$, and $5.5 \mathrm{~m} M$ glucose) supplemented with HEPES $10 \mathrm{mM}(\mathrm{pH}=7.4)$ and gentamicin solution $(50 \mu \mathrm{L} / \mathrm{L})$ at $37^{\circ} \mathrm{C}$. Once in the laboratory, $500 \mathrm{mg}$ of AT was washed 3 times in KRBB and minced in 2- to $3-\mathrm{mm}$ sections in $5 \mathrm{~mL}$ of collagenase type II solution $(2 \mathrm{mg} / \mathrm{mL}$; Worthington Biochemical, Lakewood, NJ) in KRBB with $4 \%$ BSA (Millipore, Billerica, MA). Samples were incubated in a $37^{\circ} \mathrm{C}$ water bath with inversion of the vials every 5 min for $15 \mathrm{~min}$ and then transferred to an incubator for further digestion with shaking for $45 \mathrm{~min}$ at $37^{\circ} \mathrm{C}$ and $230 \mathrm{rpm}$. Digested samples were centrifuged for $10 \mathrm{~min}$ at $300 \times g$ at room temperature to separate primary adipocytes (upper layer) from SVF (bottom layer). Primary mature adipocytes were filtered through a $250-\mu \mathrm{m}$ mesh and washed in $5 \mathrm{~mL}$ of $\mathrm{KRBB}$ with $4 \%$ BSA, and then centrifuged for $5 \mathrm{~min}$ at $300 \times g$ at room temperature. Final primary adipocyte population was retained for use in $\mathrm{CC}$ and its viability was measured by Vybrant MTT Cell Proliferation Assay Kit (Thermo
Fisher, Waltham, MA) and Cellometer ViaStain AOPI Staining in a Cellometer Vision CBA (Nexcelom Bioscience, Lawrence, MA) at d 0 and 5 after induction. The SVF was sequentially filtered through 100 - and $40-\mu \mathrm{m}$ cell strainers (Falcon, Corning, NY) and centrifuged for 10 min at $800 \times g$ at room temperature. The resulting cell pellet was resuspended and incubated in erythrocyte lysis buffer for 5 min at room temperature. Resultant cells were resuspended in $9 \mathrm{~mL}$ of basal medium containing Dulbecco's modified Eagle's medium: F12 (DMEM:F12, Corning, Corning, NY), 10\% fetal bovine serum (Corning), $2 \mathrm{mmol} / \mathrm{L}$ of L-glutamine (Corning), 1\% (vol/vol) antibiotic-antimycotic (Corning), $44.05 \mathrm{mmol} / \mathrm{L}$ of sodium bicarbonate (SigmaAldrich, St. Louis, MO), $100 \mu \mathrm{mol} / \mathrm{L}$ of ascorbic acid (Sigma-Aldrich), $33 \mu \mathrm{mol} / \mathrm{L}$ of biotin (Sigma-Aldrich), $17 \mu \mathrm{mol} / \mathrm{L}$ of pantothenate (Sigma-Aldrich), and 20 $\mathrm{mmol} / \mathrm{L}$ of HEPES (Corning), and incubated at $37^{\circ} \mathrm{C}$ in a humidified atmosphere of $95 \%$ air and $5 \% \mathrm{CO}_{2}$. Preadipocytes were obtained by outgrowth of plastic adherent cells from the SVF cells after 2 serial passages in culture flasks (Corning). Growth medium was replaced every 2 d (Strieder-Barboza et al., 2018). Confluent preadipocytes were induced to differentiate with basal medium supplemented with $5 \mu M$ troglitazone (Cayman Chemical, Ann Arbor, MI), $0.5 \mathrm{mmol} / \mathrm{L}$ of isobutyl-1-methylaxanthine (AdipoGen Life Sciences, San Diego, CA), and the following reagents from Sigma-Aldrich: $5 \mu \mathrm{g} / \mathrm{mL}$ of insulin, $10 \mathrm{~m} M$ acetate, and 1 $\mu \mathrm{mol} / \mathrm{L}$ of dexamethasone. Isobutyl-1-methylaxanthine and dexamethasone were used only during the first 48 $\mathrm{h}$ of adipogenic induction.

For CC, a 7-d differentiation protocol (CC7) was used as recently described by our laboratory (Thelen et al., 2018). Briefly, freshly isolated mature adipocytes $\left(900 \mathrm{cell} / \mathrm{cm}^{2}\right)$ were added to $0.4-\mu \mathrm{m}$ transwell inserts (Greiner Bio-One, Kremsmünster, Austria) in 24- or 12-well plates, generating a CC system between attached preadipocytes and mature adipocytes in the inserts for the first $5 \mathrm{~d}$ (out of $7 \mathrm{~d}$ ) of differentiation. After removing inserts, attached cells were kept in adipocyte differentiation medium for 2 extra days. For standard differentiation, preadipocytes were induced to differentiate using the same medium as for $\mathrm{CC} 7$ during 7 (SD7) or $14 \mathrm{~d}$ (SD14). The SD7 served as a control for lipid accumulation and lipolysis assays, whereas undifferentiated preadipocytes served as a control for PCR analysis.

We evaluated expression of the main genes involved on the transcriptional regulation of adipogenesis, such as CCAAT/enhancer-binding protein $\beta$ and $\alpha(C E B$ $P B, C E B P A), P P A R G$, and adiponectin (ADIPOQ; Ref. Seq. NM_174742.2); FA uptake and transport, such as alpha 2-HS glycoprotein/fetuin-A (AHSG), 
fatty acid translocase (CD36), fatty acid transporter-1 (FATP1), and fatty acid binding protein 4 (FABP4); and intracellular lipid synthesis, such as diacylglycerol O-acyltransferase-1 and -2 (DGAT1, DGAT2), glycerol3-phosphate acyltransferase-1 and -2 (GPAT1, GPAT2), and lipin 1/phosphatidate phosphatase (LPIN1). The RNA extraction, purification, conversion to cDNA, and quantitative PCR analysis were performed as described previously (Contreras et al., 2017). The RNA samples had an average integrity number of $8.65 \pm 1.26$ (mean \pm SD) as evaluated by an Agilent 2100 Bioanalyzer (Agilent Technologies, Santa Clara, CA). The most stable reference genes, eukaryotic translation initiation factor 3 subunit K (EIF3K), 50S ribosomal protein L15 (RPLO), and 40S ribosomal protein S9 (RPS9), were selected using geNorm (Vandesompele et al., 2002). The cycle threshold $(\mathbf{C t})$ values were converted to normalized relative gene expression as described by Hellemans et al. (2007). Briefly, each sample's duplicate Ct and efficiency (E) values were averaged, and $\mathrm{Ct}$ values of all samples within the same gene were averaged (Ctall) to be used in $2^{(-\Delta \mathrm{Ct})}$ calculations $\left\{2^{(-\Delta \mathrm{Ct})}=\mathrm{E}^{[-1 \times(\mathrm{Ct}-\mathrm{Ctall})]}\right\}$. Thus, $2^{(-\Delta \Delta \mathrm{Ct})}$ was calculated by the ratio of $2^{(-\Delta \mathrm{Ct})}$ of target genes and the geometric mean of reference genes' $2^{(-\Delta \mathrm{Ct})}$. Triacylglycerol accumulation was examined by AdipoRed (Lonza, Allendale, NJ) and HCS LipidTox staining (Life Technologies, Carlsbad, CA) by confocal microscopy imaging in an Olympus FluoView 1000 Confocal Laser Scanning Microscope (Olympus America Inc., Center Valley, PA) configured on an IX81 inverted microscope and FV10-ASW software (version 4.2.3.6; Olympus America Inc.) using a UPLFLN $20 \times / 0.50$ dry objective. Relative fluorescence units were corrected by number of cells. Responses to $\beta$-adrenergic stimulation were evaluated by glycerol release in the supernatant (cat. no. MAK117-1KT, Millipore) after a 2-h stimulation of adipocytes with $1 \mu M$ isoproterenol (ISO; Sigma-Aldrich) and corrected by number of cells. Cells were serum-starved with 0.1\% BSA-DMEM/F12 medium for $4 \mathrm{~h}$ prior to lipolysis induction. Media alone [KRBB-HEPES containing 3\% FA free BSA (Millipore)] served as negative control (CON). Data were analyzed using JMP Statistical Software (SAS Institute Inc., Cary, NC). Normality of the variables was checked using the Kolmogorov-Smirnov test $(P<0.05)$. Nonnormally distributed variables were natural log transformed. One-way ANOVA pairwise comparisons were performed using the Tukey's post hoc test. Results are presented as mean \pm SEM unless otherwise stated. Mean differences were considered significant when $P \leq$ 0.05 and tendencies when $P<0.10$.

Ours is the first study to evaluate the adipogenic efficiency of a CC model on VIS and SC SVF-derived preadipocytes. Co-culture methods have previously been used in the differentiation of human SC adipocytes (Considine et al., 1996; Janke et al., 2002; Stacey et al., 2009) and with perivascular, gonadal, and mesenteric adipocytes from rats (Thelen et al., 2018). In the present study, the viability of primary adipocytes in transwell inserts decreased from $85.7 \pm 3.9$ to $68.3 \pm$ $0.35 \%$ on d 5 after adipogenic induction of attached preadipocytes $(P<0.05)$. By the end of the differentiation protocols, $\mathrm{CC} 7$ and SD14 increased the gene expression of $A D I P O Q, C E B P A, C E B P B$, and $A H S G$ compared with undifferentiated preadipocytes $(P<0.05$; Figure 1 and Figure 2). Compared with undifferentiated preadipocytes, CC7 and SD14 augmented the gene expression of the FA transporter FABP4 in SC and that of $L P I N 1$, a required lipogenic enzyme for TAG synthesis in adipocytes, in VIS $(P<0.05)$. Whereas $C E B P B$ and $C E B P A$ expression directs the adipocyte differentiation process, expression of $A D I P O Q, F A B P 4, A H S G$, and LPIN1 promotes FA transport and TAG accumulation; therefore, these genes are highly expressed in lipidfilled mature adipocytes (Arimochi et al., 2016). Our results demonstrate that, in a shorter period of time, CC7 efficiently stimulated the transcription of critical adipogenesis and lipogenesis regulators in both SC and VIS cells, as induced by SD14 protocol.

The hallmark of adipogenesis is the mitotic arrest and the beginning of biosynthesis of specialized proteins required for cytoplasmic accumulation of TAG in lipid droplets (MacDougald and Mandrup, 2002). We measured cytoplasmic TAG accumulation in adipocytes. Even though CC7 and SD14 had similar TAG accumulation, CC7 synthesized similar amounts of cytoplasmic TAG in the half of time of SD14 in both VIS and SC $(P>0.05$; Figure 3 and Figure 4$)$. Similar evidence was previously described in SC human adipocytes in CC conditions (Stacey et al., 2009). These results suggest that the presence of mature adipocytes during CC may aid in the differentiation of adjacent cells enhancing lipogenesis (Considine et al., 1996).

As important as the capacity of synthesizing and accumulating TAG is the ability of responding to lipolytic stimulus to release glycerol and FA during periods of negative energy balance. In SC, responsiveness to $\beta$-adrenergic stimulation was higher in CC7 and SD14 compared with SD7, as demonstrated by higher glycerol release in ISO $(P<0.05$; Figure 5A). Glycerol release by SC was similar between $\mathrm{CON}$ and ISO in SD7 $(P>$ $0.05)$ and higher in ISO than CON in CC7 and SD14 ( $P$ $=0.02)$. Upon ISO stimulation, VIS cells released similar amounts of glycerol between SD7, SD14, and CC7 $(P>0.05$; Figure 5B). Compared with CON, glycerol release by VIS stimulated with ISO was similar in SD7 

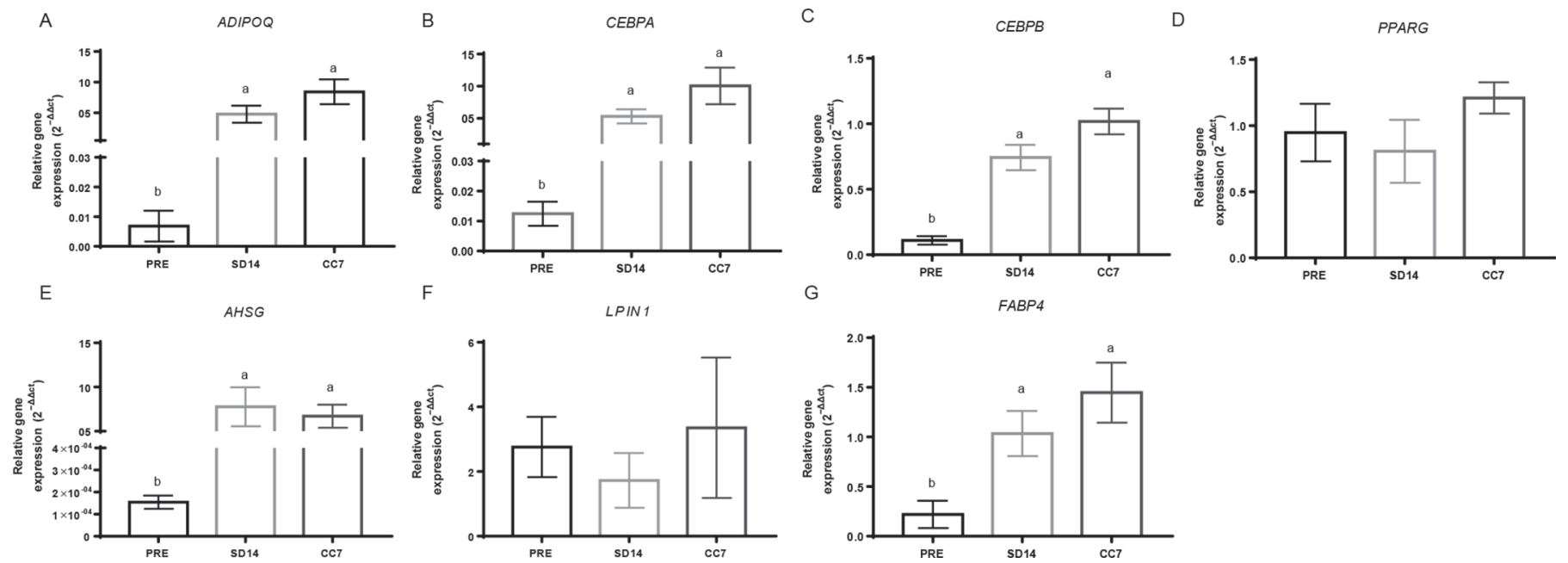

Figure 1. Gene expression of adipogenic and lipogenic markers in subcutaneous bovine adipocytes cultured with a standard differentiation protocol of $14 \mathrm{~d}$ (SD14) or using a co-culture model (CC7). Undifferentiated preadipocytes (PRE) from subcutaneous adipose tissue depot were used as a negative control. The relative gene expression of $(\mathrm{A})$ adiponectin $(A D I P O Q)$, (B) CCAAT/enhancer-binding protein $\alpha(C E B P A)$, (C) CCAAT/enhancer-binding protein $\beta(C E B P B),(\mathrm{D})$ peroxisome proliferator-activated receptor gamma $(P P A R G),(\mathrm{E})$ fetuin-A $(A H S G),(\mathrm{F})$ phosphatidate phosphatase (LPIN1), and $(\mathrm{G})$ fatty acid binding protein-4 (FABP4) were normalized to gene expression of control genes 40S ribosomal protein S9 (RPS9), eukaryotic translation initiation factor 3 subunit K (EIF3K), and 50S ribosomal protein L15 (RPLO).Values are shown as $2^{(-\Delta \Delta \mathrm{Ct})}$ (where $\mathrm{Ct}=$ cycle threshold). Results represent means \pm SEM. Bars with different letters $(\mathrm{a}, \mathrm{b})$ are significantly different $(P$ $\leq 0.05)$.

$(P>0.05)$, tended to increase in $\mathrm{SD} 14(P=0.10)$, and was higher in $\mathrm{CC} 7(P=0.02)$. Notably, although SD7 and SD14 responses to lipolysis stimulation were similar in VIS $(P>0.05)$, CC7 had greater responsiveness than SD7 $(P<0.05$, Figure 5B). These results suggest that $\mathrm{CC} 7$ may be a suitable model for evaluating lipolytic responses and pathways in visceral AT. This is particularly important because many in vitro studies
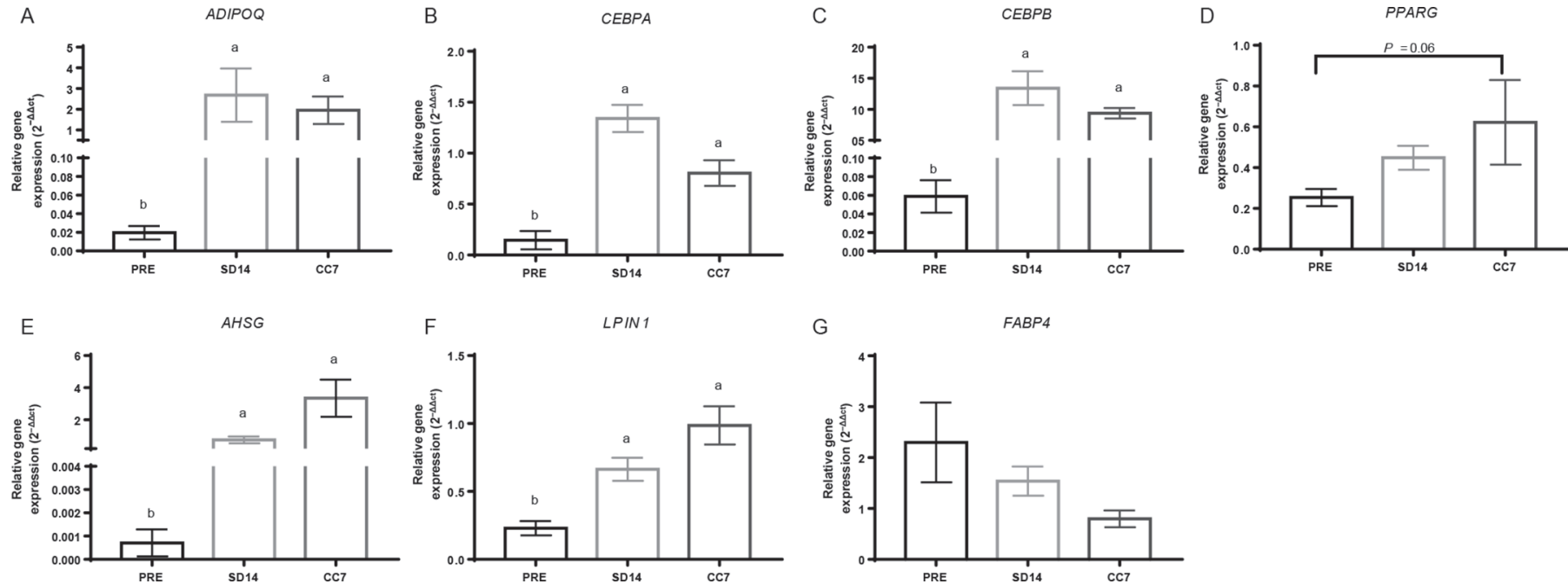

Figure 2. Gene expression of adipogenic and lipogenic markers in visceral bovine adipocytes cultured with a standard differentiation protocol of $14 \mathrm{~d}$ (SD14) or using a co-culture model (CC7). Undifferentiated preadipocytes (PRE) from omental visceral adipose tissue depot were used as a negative control. The relative gene expression of $(\mathrm{A})$ adiponectin $(A D I P O Q)$, (B) CCAAT/enhancer-binding protein $\alpha(C E B P A),(\mathrm{C})$ CCAAT/enhancer-binding protein $\beta(C E B P B)$, (D) peroxisome proliferator-activated receptor gamma $(P P A R G),(\mathrm{E})$ fetuin-A $(A H S G)$, (F) phosphatidate phosphatase ( LPIN1), and $(\mathrm{G})$ fatty acid binding protein-4 (FABP4) were normalized to gene expression of control genes $40 \mathrm{~S}$ ribosomal protein S9 (RPS9), eukaryotic translation initiation factor 3 subunit $\mathrm{K}$ (EIF3K), and $50 \mathrm{~S}$ ribosomal protein L15 (RPLO).Values are shown as $2^{(-\Delta \Delta \mathrm{Ct})}$ (where $\mathrm{Ct}=$ cycle threshold). Results represent means \pm SEM. Bars with different letters $(\mathrm{a}, \mathrm{b})$ are significantly different $(P$ $\leq 0.05)$. 

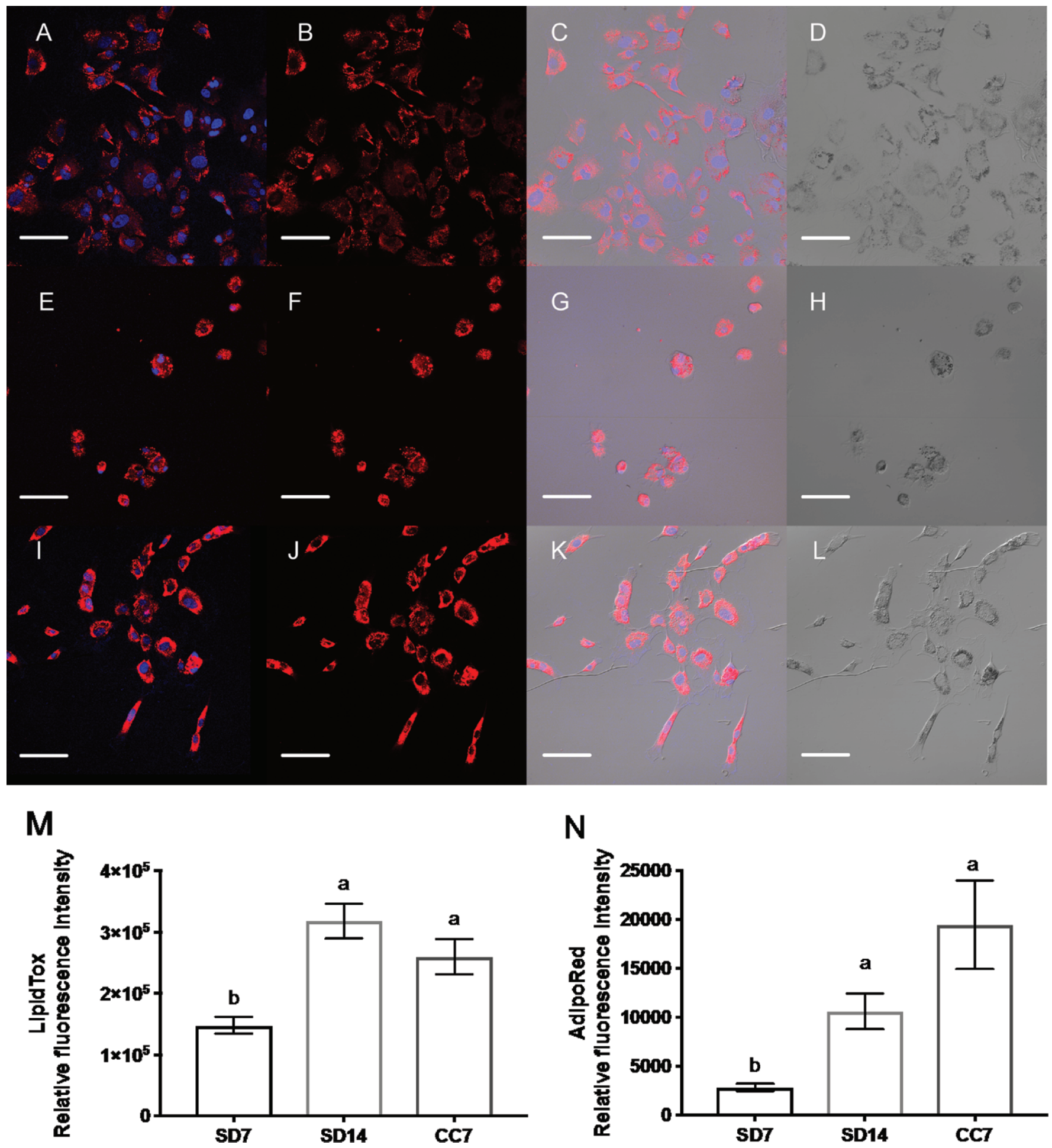

Figure 3. Lipid accumulation in cultured bovine adipocytes derived from subcutaneous adipose tissue in dairy cows. (A-L) Laser scanning confocal microscopy imaging of lipid droplets in bovine adipocytes at the last day of standard differentiation for $7 \mathrm{~d}$ (SD7; A-D) co-culture differentiation for $7 \mathrm{~d}(\mathrm{CC} 7 ; \mathrm{E}-\mathrm{H})$ and standard differentiation for $14 \mathrm{~d}$ (SD14; I-L). (A, E, I) Adipocytes co-stained with the lipid droplet stain HCS LipidTox (red fluorescence; Life Technologies, Carlsbad, CA) and nuclei stain NucBlue (blue fluorescence; Life Technologies). (B, F, J) Bovine adipocytes stained with HCS LipidTox red fluorescence. (C, G, K) Differential interference contrast images of bovine adipocytes costained with HCS LipidTox and NucBlue. (D, H, L) Differential interference contrast images of bovine adipocytes. (M) Graphical representation (means \pm SEM) of relative fluorescence intensity measured through confocal microscopy images using ImageJ software (https://imagej.nih.gov/ $\mathrm{ij} /$ ). (N) Relative fluorescence intensity (means \pm SEM) obtained by AdipoRed assay (Lonza, Allendale, NJ). Bars with different letters (a-c) are significantly different $(P \leq 0.05)$. Scale bars $=100 \mu \mathrm{m}$. 


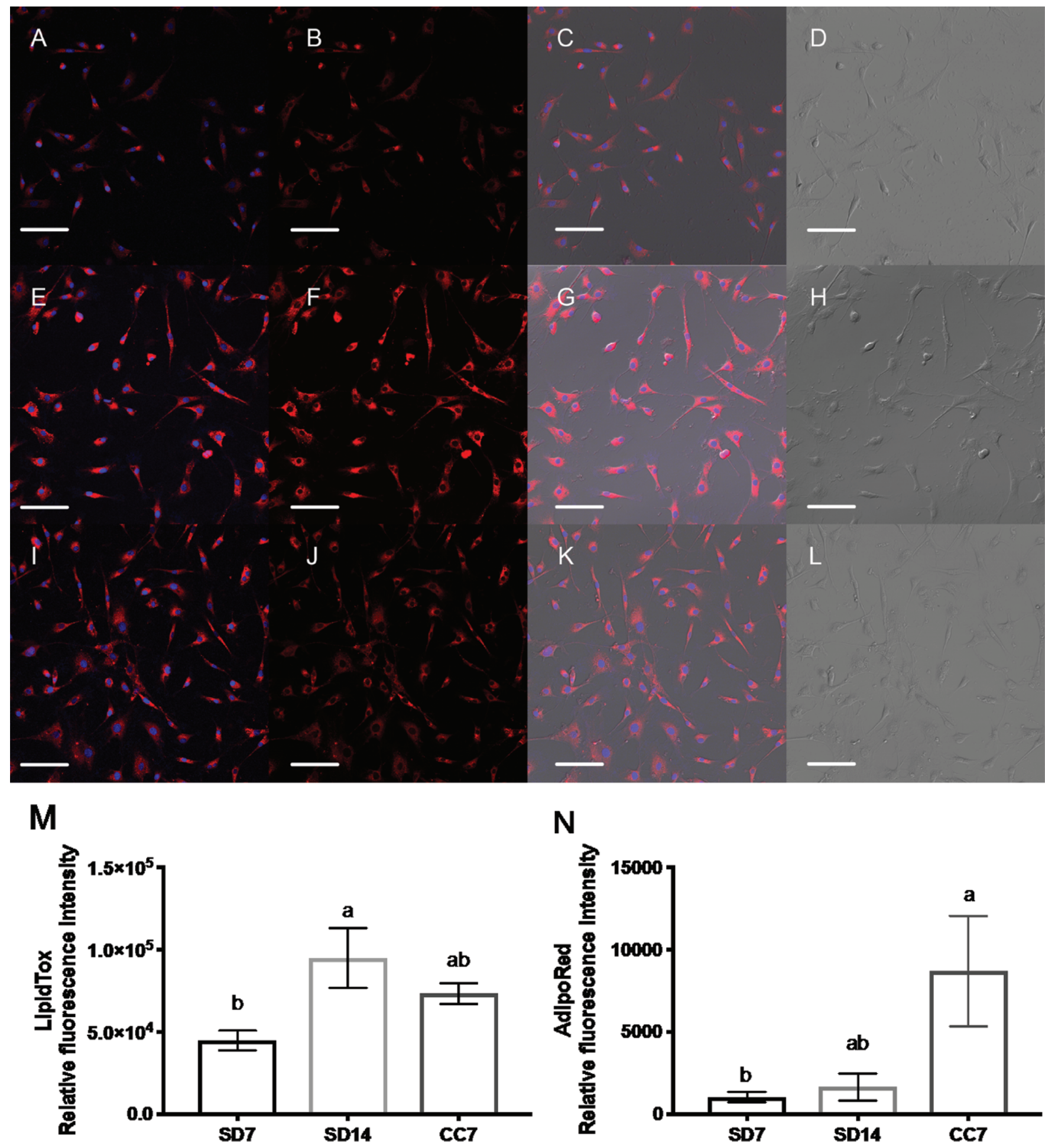

Figure 4. Lipid accumulation in cultured bovine adipocytes derived from visceral adipose tissue in dairy cows. (A-L) Laser scanning confocal microscopy imaging of lipid droplets in bovine adipocytes at the last day of standard differentiation for $7 \mathrm{~d}$ (SD7; A-D), co-culture differentiation for $7 \mathrm{~d}(\mathrm{CC} 7 ; \mathrm{E}-\mathrm{H})$, and standard differentiation for $14 \mathrm{~d}$ (SD14; I-L). (A, E, I) Adipocytes co-stained with the lipid droplet stain HCS LipidTox (red fluorescence; Life Technologies, Carlsbad, CA) and nuclei stain NucBlue (blue fluorescence; Life Technologies). (B, F, J) Bovine adipocytes stained with HCS LipidTox red fluorescence. (C, G, K) Differential interference contrast images of bovine adipocytes co-stained with HCS LipidTox and NucBlue. (D, H, L) Differential interference contrast images of bovine adipocytes. (M) Graphical representation (means \pm $\mathrm{SEM}$ ) of relative fluorescence intensity measured through confocal microscopy images using ImageJ software (https://imagej.nih.gov/ij/). (N) Relative fluorescence intensity (means \pm SEM) obtained by AdipoRed assay (Lonza, Allendale, NJ). Bars with different letters (a-c) are significantly different $(P \leq 0.05)$. Scale bars $=100 \mu \mathrm{m}$. 

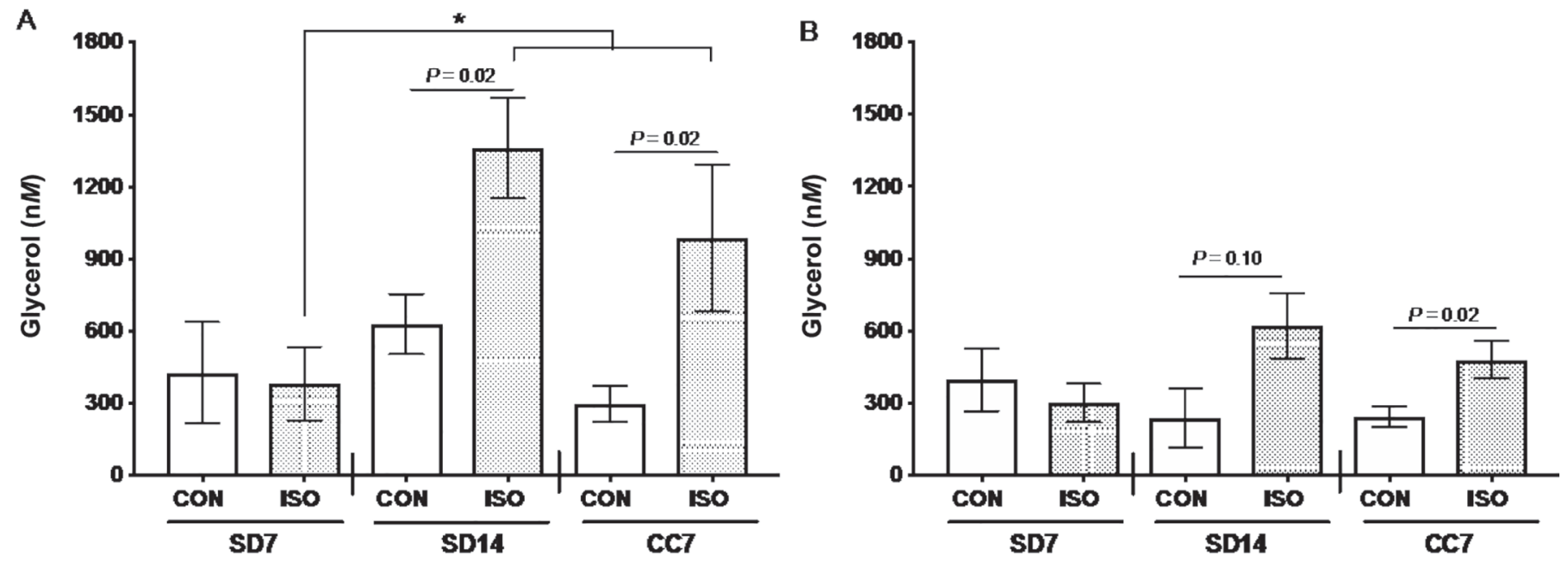

Figure 5. Responsiveness of subcutaneous and visceral adipocytes differentiated in standard or co-culture conditions to $\beta$-adrenergic stimulation. Concentrations of glycerol (nanomolar) released in the medium during 2-h stimulation with (ISO) or without (CON) isoproterenol (1 $\mu M)$ in (A) subcutaneous and (B) visceral adipocytes. Cells were induced to differentiate using a standard protocol for 7 (SD7; control) or 14 d $(\mathrm{SD} 14)$, and a co-culture $(\mathrm{CC} 7)$ in vitro model. ${ }^{*} P<0.05$. Values represent means $\pm \mathrm{SEM}$.

have been performed with only $\mathrm{SC}$ adipocytes due to the low adipogenic efficiency of visceral SFV, potentially missing visceral-specific regulatory mechanisms and differential physiological functions compared with other AT depots (Macotela et al., 2012).

Even though such CC seems be an efficient model for adipogenesis induction and one step closer to mimicking the in vivo AT environment, it also has limitations. Collecting primary adipocytes from abattoirs is laborious and may not be practical for all types of research settings. However, the increased adipogenic efficiency in a short 7-d protocol, especially in VIS, may offset the limitations of the protocol. Our CC7 model also has potentially great value for experiments with genetic manipulation of cells (i.e., CRISPR) in which a maximum adipogenic efficiency is required. Future studies will test CC models in more feasible and functional settings, such as the replacement of the primary mature adipocytes from abattoir by in vitro differentiated adipocytes.

In the current study, we demonstrated that a 7-d CC protocol with VIS or SC bovine adipocytes improved adipogenic efficiency by promoting similar responses to a 14-d standard differentiation protocol in a shorter period. The co-culture model is suitable for studying VIS adipocyte functions and may partially mimic the complex differentiation stimuli provided in vivo. Co-culture of preadipocytes with mature adipocytes is physiologically relevant for the study of adipocyte biology and the assessment of pharmacological or nutritional interventions in reductionist models with bovine adipocytes.

\section{ACKNOWLEDGMENTS}

This research was funded by USDA-National Institute of Food and Agriculture (Washington, DC) grants 2015-67015-23207 and the Department of Large Animal Clinical Sciences (Michigan State University, East Lansing). The authors are grateful to Jennifer Dominguez and the staff at the Michigan State University Meat Laboratory (East Lansing) and West Michigan Beef (Hudsonville, MI) for providing samples.

\section{REFERENCES}

Arimochi, H., Y. Sasaki, A. Kitamura, and K. Yasutomo. 2016. Differentiation of preadipocytes and mature adipocytes requires PSMB8. Sci. Rep. 6:26791.

Considine, R. V., M. R. Nyce, L. M. Morales, S. A. Magosin, M. K. Sinha, T. L. Bauer, E. L. Rosato, J. Colberg, and J. F. Caro. 1996. Paracrine stimulation of preadipocyte-enriched cell cultures by mature adipocytes. Am. J. Physiol. 270:E895-E899.

Contreras, G. A., C. Strieder-Barboza, and J. De Koster. 2018. Symposium review: Modulating adipose tissue lipolysis and remodeling to improve immune function during the transition period and early lactation of dairy cows. J. Dairy Sci. 101:2737-2752.

Contreras, G. A., C. Strieder-Barboza, J. de Souza, J. Gandy, V. Mavangira, A. L. Lock, and L. M. Sordillo. 2017. Periparturient lipolysis and oxylipid biosynthesis in bovine adipose tissues. PLoS One 12:e0188621.

Grant, A. C., G. Ortiz-Colon, M. E. Doumit, and D. D. Buskirk. 2008. Optimization of in vitro conditions for bovine subcutaneous and intramuscular preadipocyte differentiation. J. Anim. Sci. 86:73-82.

Hellemans, J., G. Mortier, A. De Paepe, F. Speleman, and J. Vandesompele. 2007. qBase relative quantification framework and software for management and automated analysis of real-time quantitative PCR data. Genome Biol. 8:R19.

Janke, J., S. Engeli, K. Gorzelniak, F. C. Luft, and A. M. Sharma. 2002. Mature adipocytes inhibit in vitro differentiation of human preadipocytes via angiotensin type 1 receptors. Diabetes 51:16991707. 
Lengi, A. J., and B. A. Corl. 2010. Factors influencing the differentiation of bovine preadipocytes in vitro. J. Anim. Sci. 88:1999-2008.

MacDougald, O. A., and S. Mandrup. 2002. Adipogenesis: Forces that tip the scales. Trends Endocrinol. Metab. 13:5-11.

Macotela, Y., B. Emanuelli, M. A. Mori, S. Gesta, T. J. Schulz, Y.-H. Tseng, and C. R. Kahn. 2012. Intrinsic differences in adipocyte precursor cells from different white fat depots. Diabetes 61:16911699.

Picot, J., V. Harmelen, T. Skurk, and H. Hauner. 2005. Primary culture and differentiation of human adipocyte precursor cells. Pages 125-135 in Human Cell Culture Protocols. Vol. 107. Humana Press, New York, NY.

Smith, S., H. Kawachi, C. Choi, C. Choi, G. Wu, and J. Sawyer. 2009. Cellular regulation of bovine intramuscular adipose tissue development and composition. J. Anim. Sci. 87(E-Suppl.):E72-E82.

Stacey, D. H., S. E. Hanson, G. Lahvis, K. A. Gutowski, and K. S. Masters. 2009. In vitro adipogenic differentiation of preadipocytes varies with differentiation stimulus, culture dimensionality, and scaffold composition. Tissue Eng. Part A 15:3389-3399.

Strieder-Barboza, C., J. de Souza, W. Raphael, A. L. Lock, and G. A. Contreras. 2018. Fetuin-A: A negative acute-phase protein linked to adipose tissue function in periparturient dairy cows. J. Dairy Sci. 101:2602-2616.

Sugihara, H., N. Yonemitsu, S. Miyabara, and S. Toda. 1987. Proliferation of unilocular fat cells in the primary culture. J. Lipid Res. 28:1038-1045.

Thelen, K., S. W. Watts, and G. A. Contreras. 2018. Adipogenic potential of perivascular adipose tissue preadipocytes is improved by coculture with primary adipocytes. Cytotechnology 70:1435-1445.

Vandesompele, J., K. De Preter, F. Pattyn, B. Poppe, N. Van Roy, A. De Paepe, and F. Speleman. 2002. Accurate normalization of real-time quantitative RT-PCR data by geometric averaging of multiple internal control genes. Genome Biol. 3:H0034. 